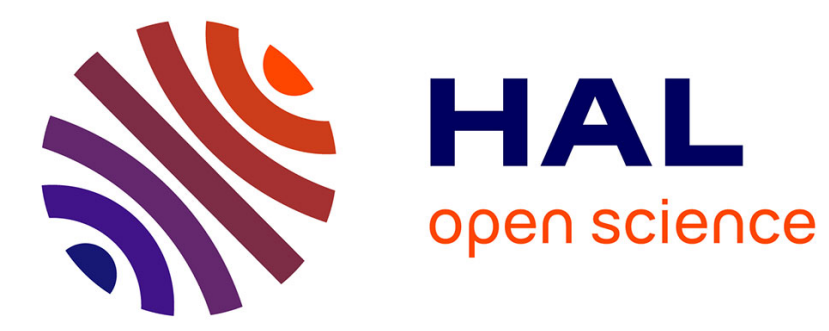

\title{
Deep recurrent neural network-based autoencoder for photoplethysmogram artifacts filtering
}

Joseph Azar, Abdallah Makhoul, Raphael Couturier, Jacques Demerjian

\section{To cite this version:}

Joseph Azar, Abdallah Makhoul, Raphael Couturier, Jacques Demerjian. Deep recurrent neural network-based autoencoder for photoplethysmogram artifacts filtering. Computers and Electrical Engineering, 2021, 92, pp.107065. hal-03186560

\section{HAL Id: hal-03186560 https://hal.science/hal-03186560}

Submitted on 31 Mar 2021

HAL is a multi-disciplinary open access archive for the deposit and dissemination of scientific research documents, whether they are published or not. The documents may come from teaching and research institutions in France or abroad, or from public or private research centers.
L'archive ouverte pluridisciplinaire HAL, est destinée au dépôt et à la diffusion de documents scientifiques de niveau recherche, publiés ou non, émanant des établissements d'enseignement et de recherche français ou étrangers, des laboratoires publics ou privés. 


\title{
Deep Recurrent Neural Network-based Autoencoder for Photoplethysmogram Artifacts Filtering
}

\author{
Joseph Azar ${ }^{\mathrm{a}, *}$, Abdallah Makhoul ${ }^{\mathrm{a}}$, Raphaël Couturier ${ }^{\mathrm{a}}$, Jacques Demerjian ${ }^{\mathrm{b}}$ \\ ${ }^{a}$ FEMTO-ST Institute, UMR 6174 CNRS, Univ. Bourgogne Franche-Comté, France \\ ${ }^{b}$ LaRRIS, Faculty of Sciences, Lebanese University, Fanar, Lebanon
}

\begin{abstract}
Recently, the need for fast, cost-effective, convenient and non-invasive cardiovascular analysis techniques is the primary attractive reason to use photoplethysmogram (PPG). Most wearable devices on the market today, are capable of collecting PPG data and enabling the measurement of important features such as heart rate, respiration rate, and blood pressure in addition to detecting irregular pulses and cardiovascular diseases. One major drawback of PPG data is its high sensitivity to motion, resulting in distorted signal and meaningless data zones. This paper proposes a neural network-based filtering method to remove corrupted zones from the collected PPG data in an unsupervised manner. It also proposes a PPG data summarization and augmentation strategy which optimizes the network performance. Experimental results show that the proposed approach was capable of achieving 90\% precision and $95 \%$ recall when processing PPG data collected from a Shimmer3 GSR+ sensor.

Keywords: Wearable-based applications, Deep learning, Anomaly detection, Photoplethysmography, Health monitoring applications

\footnotetext{
* Corresponding author abdallah.makhoul@univ-fcomte.fr (Abdallah Makhoul), raphael.couturier@univ-fcomte.fr (Raphaël Couturier), jacques.demerjian@ul.edu.lb (Jacques Demerjian)
}

Email addresses: joseph.azar@univ-fcomte.fr (Joseph Azar),
\end{abstract}




\section{Introduction}

Wearable devices and fitness tracking applications have been introduced and used by millions of people, allowing continuous and unobtrusive tracking of an individual behavior and physiological features. One of the important signals to calculate the physiological features from these devices is the photoplethysmogram (PPG) signal. Photoplethysmography (PPG) is a non-invasive optical method to monitor vital signs of the body such as heart rate, heart rate variability, and blood oxygenation. The PPG waveform represents variations in blood volume and contains important characteristics such as cycle period, baseline, and amplitude that are useful for analysis. Fortunately, wearable health monitoring devices, including smart watches and fitness trackers, can now capture PPG signals and allow cardiac activity monitoring by deriving from the PPG the same R-R intervals derived from the electrocardiogram (ECG).

Due to the enormous need to track chronic diseases and monitor elderly parents, ubiquitous health monitoring applications were listed among the various categories on the wearables market as the fastest growing segments [1] [2]. Today, current wearable devices and sensors no longer focus on basic metrics for fitness tracking such as the number of steps taken, but also control essential physiological features. Commercial smartphone and wearable devices are currently able to measure a range of physiological parameters using PPG, such as the interval between successive heartbeats, respiration rate, and blood pressure [2.

One of the challenges of using PPG-based monitoring methods is the inaccuracy of the PPG signals during daily routine activities and physical exercises. This restriction is based on the fact that PPG signals are highly vulnerable to hand motion artifacts and environmental noise [3]. Examples of clean PPG and PPG with artifacts are shown in Figure 1. The estimation of heart rate variability data is strongly affected by artifacts, and the existence of these artifacts has consequences for anyone who relies on such data for higher-level analysis in addition to wasting storage space on meaningless data.

The aim of this paper is to address the artifacts in PPG data. The problem 


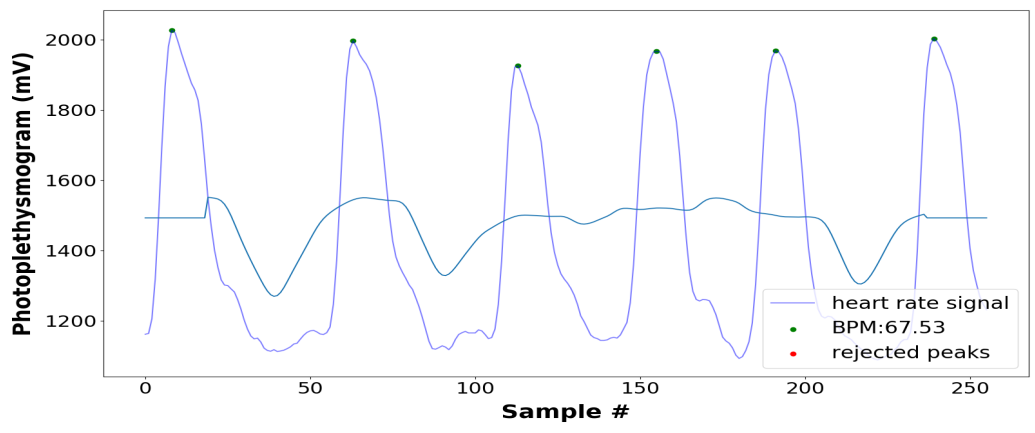

(a) clean PPG signal

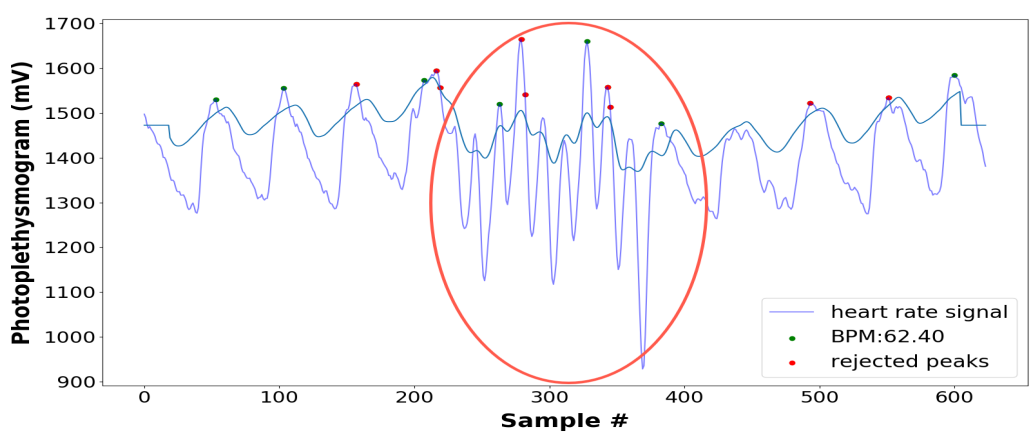

(b) PPG signal with artifacts

Figure 1: Processing two PPG signals using the HeartPy library developed in 4

can be conceived as a problem of anomaly detection since the artifacts can be viewed in a PPG signal as anomalies. The difference between denoising a PPG signal and removing artifacts is important to note. An area containing artifacts such as in Figure 1(b) is an area where features such as heart rate variability and heart rate can not be extracted, while those features can still be extracted with confidence if the signal contains an acceptable noise level.

The ability to detect anomalies in a data stream is possible with the advances of deep learning and Neural Networks (NNs). Deep learning methods like Convolution Neural Network (CNN), autoencoders, and Long Short-Term Memory (LSTM) have been commonly used for anomaly detection problems [5]. This paper proposes an unsupervised deep learning architecture based on a CNN- 
LSTM autoencoder that is able to detect artifacts in a PPG signal. Moreover, it proposes a sequences summarization approach for the neural network using the discrete wavelet transform (DWT) that enhances the training speed and and helps to avoid the problem of the vanishing gradients, as well as a data augmentation procedure for PPG data which enables a better generalization of the model.

The rest of this paper is as follows. Section 2 discusses various works based on PPG processing in wearable applications. Section 3 gives a background information on the discrete wavelet transform and explains how an autoencoder can be used for anomaly detection. Sections 4 and 5 explain the proposed data augmentation approach and deep learning architecture respectively. section 6 presents the experimental results and the conclusion is presented in section 7 .

\section{Related work}

Different research work in the literature tackled the processing and analysis of PPG data in the context of wearables and IoT. In [6], the technology of photoplethysmography and its potential applications was reviewed. This review focused on two important stages when dealing with PPG data, namely: preprocessing and feature extraction.

Van Gent et al did extensive work on analyzing and processing PPG. They developed the HeartPy library which works well with noisy PPG data 4. The developed algorithm is capable of extracting the heart rate from the raw data and estimating the breathing rate. Additionally, they proposed a method that uses the R-R intervals and R-peaks to detect error/artifacts in the data [7. In [8], the authors proposed a neural network model to enhance PPG measurements containing artifacts. The proposed approach consists of a global reference template reflecting a subject's clean heart beat morphology to be prestored in system memory. The reference template is derived through a beat quality assessment method from the acceptable quality beats of the current data. The extracted template should reflect the subject's clean heart beats morphology in 
order for the neural network to work well. In [9], the authors used frequencydomain analysis techniques such as fast Fourier transform and band-pass filtering to process PPG data. They referred to an adaptive echo cancellation method to remove the motion artifacts from the signal. The authors in [10 proposed to use the singular spectrum analysis and a spectral subtraction technique to reduce the corruption in the signal and remove the artifacts. The authors used the artifact-related 3-D accelerometer signal as an additional information to help eliminating the artifacts. Different other approaches have been proposed in [11, 12, 13, 14, 15] that use methods such as independent component analysis, Kalman smoother, wavelets, and adaptive filters in order to reduce the artifacts from PPG data.

The contribution of this paper, as compared to the above approaches, consists of detecting and removing the zones where the data are meaningless, and not denoising or enhancing the signal. The removal of these zones happens in an unsupervised manner without the need of extra information from other types of data or manually crafted features.

\section{Background}

\subsection{Discrete wavelet transform}

The Discrete Wavelet Transform (DWT) allows a signal to be represented in a time-frequency domain. It divides the signal into components of low frequency (approximations) and components of high frequency (details) by using filters as shown in Figure 2 .

The inspiration to use the DWT lives in transforming redundant samples in the temporal domain into decorrelated coefficients in the time-frequency domain, enabling the original samples to be compacted and represented with less coefficients [16 [17. This process therefore helps facilitate the analysis of certain original dataset's features.

DWT has been used in several research works for time series classification tasks [18] 19. Usually, accuracy is evaluated in accordance with other criteria 


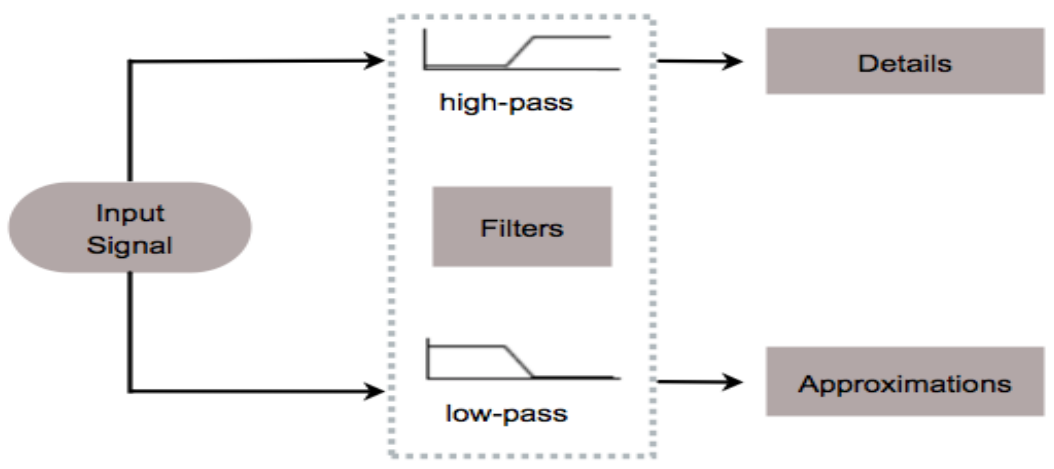

Figure 2: Discrete Wavelet Transform frequency portions of signal

when comparing several competing classification approaches. The classification algorithm's computation time (speed) is probably the second most important criterion, particularly for time series data. Since DWT produces a number of signal decompositions, the classification methods can be applied to the wavelettransformed domain at a specific level. Compared to the original data, the wavelet coefficients are sets of smaller size, and therefore the computation speed of the classification method can be increased.

\subsection{Autoencoder-based anomaly detection}

An autoencoder is a particular type of neural network which copies the input values to the output values. It consists of two modules, the encoder and the decoder. The encoder is learning a process's latent space representation. Typically, the latent features are in a smaller dimension. From these underlying features, the decoder can reconstruct the original data. The autoencoder can be used for anomaly detection problems. This is done by learning the pattern of a normal process. A given input that does not follow this pattern is then categorized as an anomaly since the model will find it different from what it has learned during the training phase. The reconstruction error is the metric used to evaluate a given input. By defining a threshold, an input vector can be labeled as anomaly if the difference between the values of this input and the output exceeds the threshold. Autoencoder-based anomaly detection can be used to 
process different types of data such as images and time series, therefore it can contains convolution layers (CNN autoencoder), long short-term memory layers (LSTM autoencoder), or a combination of both (CNN-LSTM autoencoder).

\section{Data augmentation}

The approach to data augmentation used in this paper is to apply various types of noise to the existing time series. The purpose of data augmentation is to prevent overfitting and enhance a deep learning model's generalization ability. Different approaches to data augmentation were proposed in the literature such as in 20. While working with medical signals such as PPG, the main challenge is to ensure that the signal produced follows the temporal order of samples and the original signal form/shape. In other words, the peaks should still be easy to detect and the time between two consecutive peaks should stay the same so that the same features can be derived from the newly generated data as from the original data.

\subsection{Gaussian and uniform noise}

Uniform noise has a flat distribution which ranges between 0 and 1 , which means that it is equally likely to draw all values between 0 and 1 . Normally distributed noise, or Gaussian noise, has a zero-centered "bell-curve" distribution, with most values clustered to zero. Through adding and multiplying by some constants, the distribution of both uniform and Gaussian noises can be shifted and stretched. Such constants are carefully selected to keep the produced signal analyzable and meaningful and their values depend on the application and the available data.

These two types of noise are referred to as "white noise" since they have a flat power spectrum.

\subsection{Scaling and magnitude-warping}

The scaling approach adjusts the magnitude of the data in a batch by multiplying by a random scalar, while magnitude-warping adjusts the magnitude 
of each sample in this batch by convolving the data with a smooth curve that varies around one. These two approaches were inspired from the work proposed in [20. The results of applying scaling and magnitude-warping to a PPG time series are shown in Figure 3 .
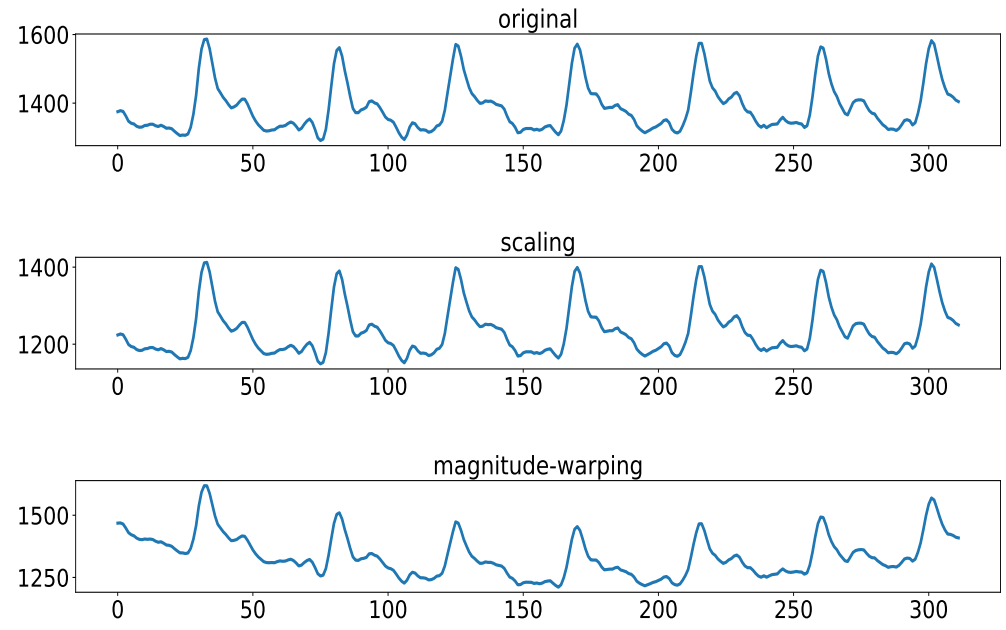

Figure 3: Applying scaling and magnitude-warping to a PPG time series

\subsection{Pink and brownian noise}

Pink noise, also known as fractal noise or $\frac{1}{f}$ noise, has a power spectrum that decreases as the frequency increases. Pink noise is weighted toward low frequencies, as its power decreases like $\frac{1}{f}$. The application of a vanishing frequency filter is one way to compute pink noise [21].

Brownian noise further lowers the higher frequencies than the pink noise. It is also known as random walk noise and is calculated by integrating random noise that is normally distributed. Brownian noise has memory, which means that its past values influence each time point. Figure 4 shows the results of adding pink and brownian noise to a PPG time series. Although the shape of the time series has been affected, it is still possible to extract the relevant features. 

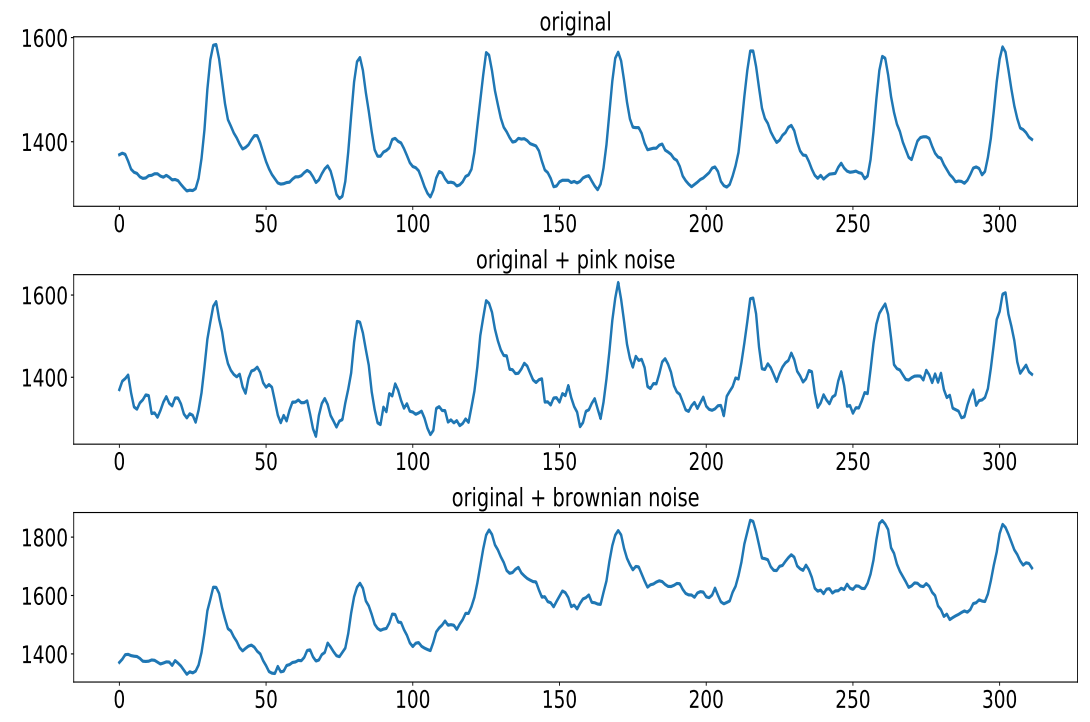

Figure 4: Adding pink and brownian noise to a PPG time series

\section{Our proposed neural network model}

In this paper, we propose the use a hybrid model, namely CNN-LSTM autoencoder, to detect the artifacts in a signal in an unsupervised manner. Given that PPG data are time series, an interesting approach is to use a model based on LSTM. If input sequences are very long, such as a PPG trace of hundreds of time steps, LSTMs can be difficult to use. The first problem of dealing with long sequences is the very long time required to train the model. Additionally, the back propagation through long input sequences can lead to vanishing gradients and, in turn, an unlearnable model. The first step in this paper's proposed solution is to address the issue of long input sequences. Note that the goal is to check that a PPG data window is clean or contains artifacts, and that window can contain hundreds to thousands of data points. The approach taken is sequences summarization using the discrete wavelet transform (DWT). In natural language processing where input sequences are words, summarizing sequences were used, it might be possible to eliminate all words from input sequences above a defined word frequency. Instead of removing samples from the data, 
the use of the DWT will introduce a more compact version of the signal to the neural network. It can be viewed as a summary of the original signal with fewer data points. For example, if we take a PPG signal with length of 312 as shown in Figure 5 applying one-level decomposition will result in a set of 156 approximation coefficients and 156 detail coefficients. Then another decomposition can be applied to the approximations obtained, resulting in 78 approximation and detail coefficients. The model will then be trained on the approximation coefficients and the details will be discarded. As a result, the speed of the learning could be improved and the problem of the vanishing gradient avoided.
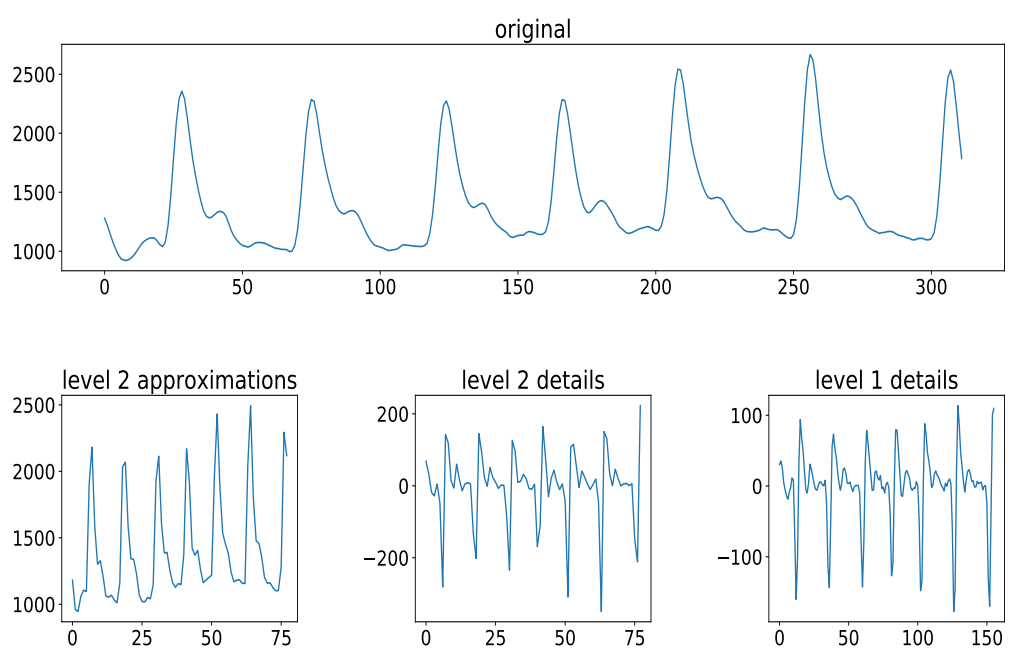

Figure 5: Example of the two-level decomposition of a PPG signal

The proposed architecture takes as inputs a fixed-length vector with shape $<S, T, 1>$, where $S$ is the number of data windows in a mini-batch, and $T$ is the number of samples in each data window. Note that the data are standardized before being initialized to the model. The encoder part is made up of a $1 \mathrm{D}$ convolution layer with a kernel size of 5 and 320 feature maps, followed by a 1D max pooling layer with a pool size of 4 and a dropout layer with a rate of $40 \%$. The output of the convolution layer is then flattened and fed into a bidirectional 
LSTM layer with 256 units followed by a second dropout layer. The decoder part consists of a LSTM layer with 192 units followed by a Gaussian noise layer for regularization and a time distributed dense layer. The linear activation function has been used for the output dense layer and ReLu [22] for the convolution and LSTM layers. Additionaly, the He normal initialization [23] was used for weights initialization, the mean squared error was used as a loss function, and Adam 24] as optimizer with gradient clipping to avoid exploding gradients. Figure 6illustrates the proposed approach used for unsupervised PPG artifacts

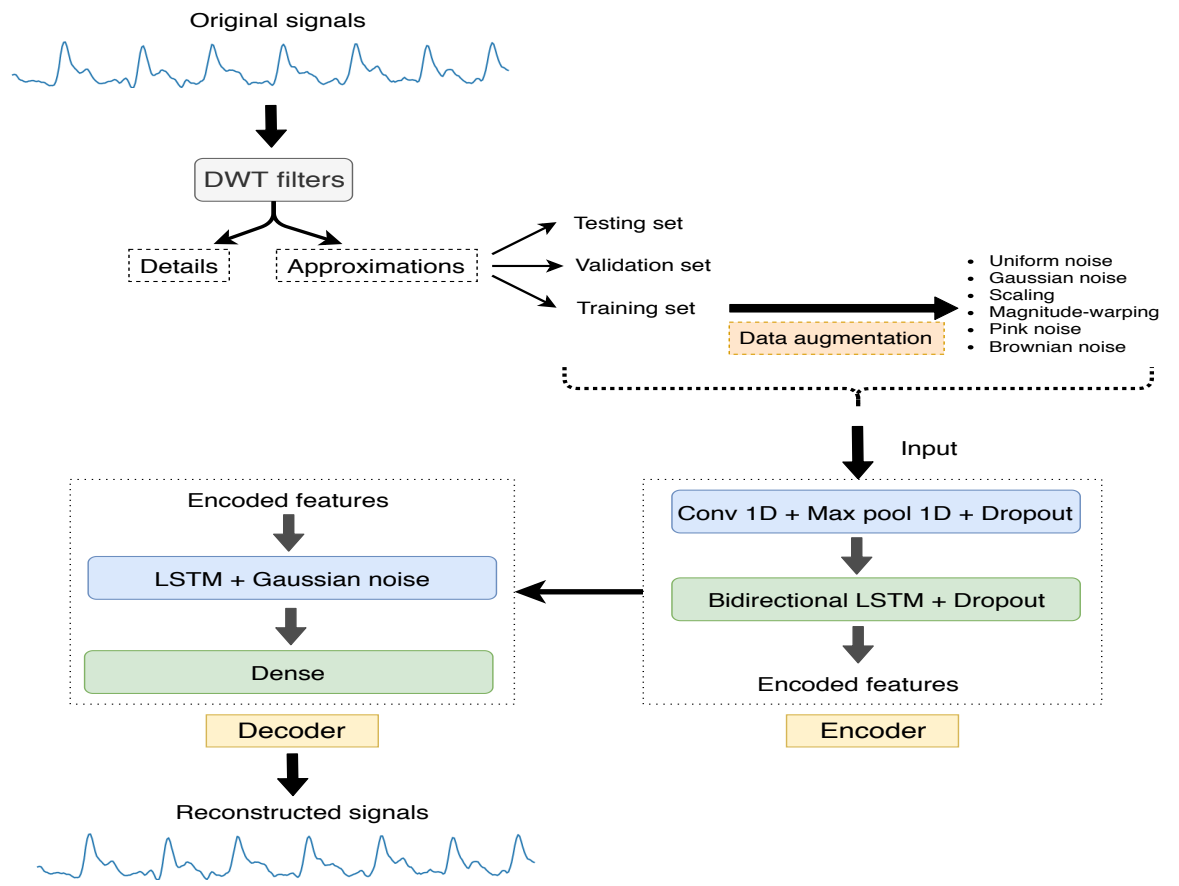

Figure 6: Proposed neural network approach for unsupervised PPG artifacts detection using CNN-LSTM Autoencoder

detection. Note that the data augmentation described in section 5 is applied on the training set after applying the wavelet transform on the original signals and splitting the resulting approximations into training, validation and testing sets. Then the model is trained only on clean PPG signals and tested on mixed PPG signals. 


\section{Experimental results}

The PPG dataset was collected using Shimmer3 GSR+Unit [25] with a sampling frequency of $F s=52 \mathrm{~Hz}$ in the Femto-ST laboratory department DISC, Belfort, France. An overlapping sliding window was used to split the collected data set into batches, resulting in a training set containing 10212 batches of clean PPG and 3149 batches of artifacts, a validation set containing 4358 batches of clean PPG and 1369 batches of artifacts, and a test set containing 150 unlabeled windows of clean PPG and artifacts.

In the experimentation two strategies are considered. The first is to use PPG recording windows of 3 seconds ( $F s \times 3=156$ samples), so the model's input will be a tensor of shape $\langle S, 156,1\rangle$. The second strategy is the proposed one, which takes larger PPG recording windows of 6 seconds (312 samples) and then applies two-level decomposition on these windows to reduce their length. As a result, the model's input will be a tensor of shape $\langle S, 78,1>$. The data augmentation process has been applied to the second strategy only in order to assess its advantage on the final output. The computations were performed on a NVIDIA Tesla Titan X GPU.

In order to determine what benefit training the model on wavelet approximations and augmenting the data give compared to the standard approach, we used the Precision vs. Recall and Receiver Operating Characteristic (ROC) curves. The ROC curve illustrates the trade-off between the true positive rate and the false positive rate for a model using different thresholds. Precision vs. Recall curve illustrates the trade-off between the true positive rate and the positive predictions for a model using different thresholds. Note that high precision is associated with a low false positive rate and high recall is associated with a

low false negative rate. The thresholds in the curves are the autoencoder reconstruction error and the desired outcome is a model with high scores for both metrics (high precision and high recall).

Figure 7 shows that a better balance between recall and precision could be achieved by the proposed approach. If the model is trained on three seconds 

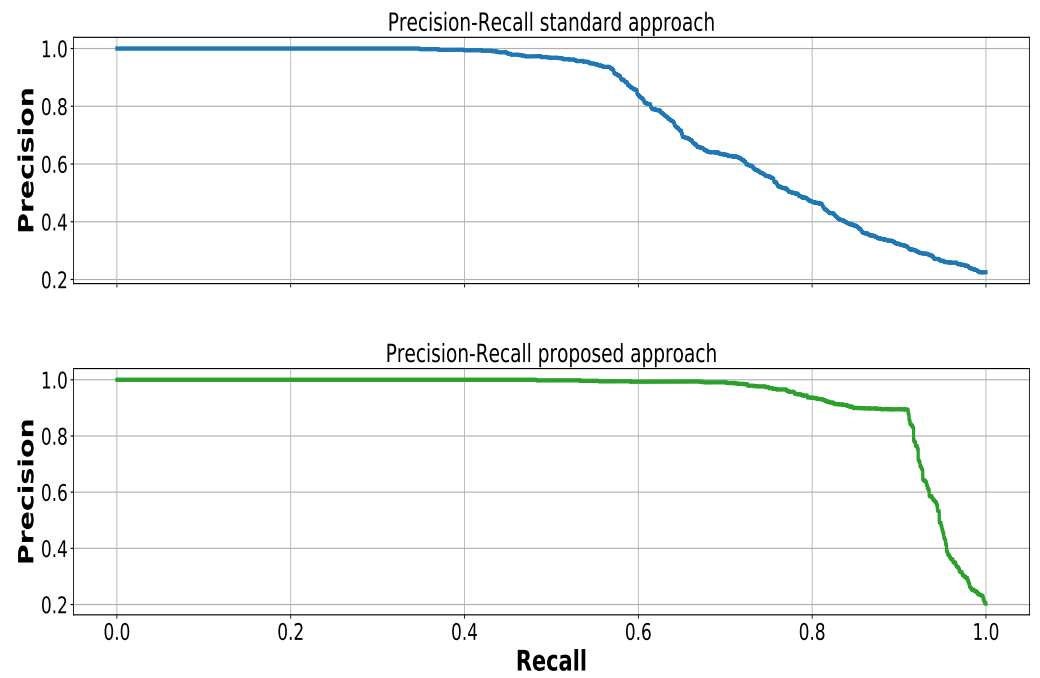

Figure 7: Precision and recall for different threshold values

PPG windows with no data augmentation, the model will have a precision of less than 0.4 in order to achieve a recall greater than 0.9 while the model was able to have a precision of 0.9 with a recall greater than 0.9 when trained on six seconds compacted PPG windows with data augmentation. During the experiments, it was noticed that the larger the data window, the easier it was for the model to detect anomalies since large PPG windows contain more features and enable the model to learn more about the regular shape of PPG signals. The improved results of the proposed approach can be explained by the fact that we can allow the model to learn the patterns and shape of larger windows of PPG data from a summarized version by using the discrete wavelet transform.

Figure 8 shows that the proposed approach have a higher area under the ROC curve (AUC) than the standard approach. Note that a model with an AUC higher than 0.5 is better than a random classifier. Selecting a threshold that gives a true positive rate greater than 0.9 and a false positive rate close to 0 is possible when using data augmentation.

In order to define whether or not a PPG signal is valid, we defined the cut 


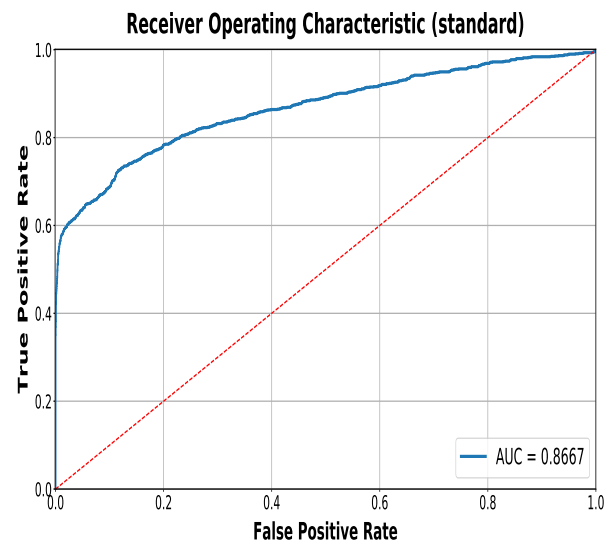

(a)

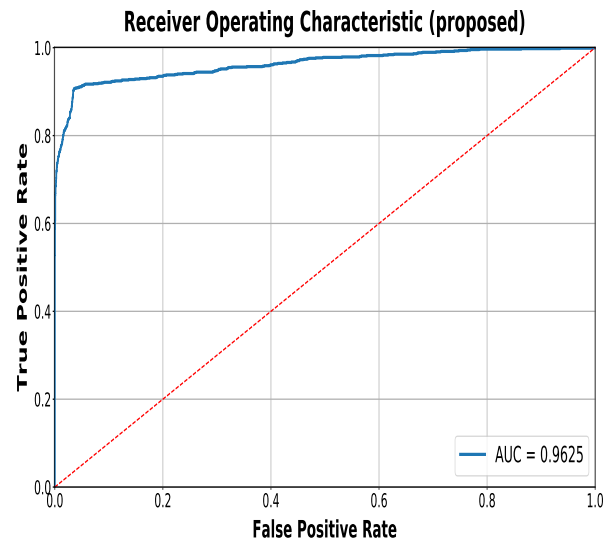

(b)

Figure 8: Receiver operating characteristic curves for the standard and proposed approaches.

point as 0.06 , this threshold is based on the last training loss value obtained during the training. If the mean squared error between the reconstructed signal and the input exceeds this threshold then this signal is marked as a meaningless PPG signal. Figure 9 shows the reconstruction of one clean PPG signal and two irrelevant signals. The mean squared error when the signal is clean is 0.02 , while it exceeds the threshold value for the other two signals. It was noticed that there was a reconstruction error greater than 0.1 in most PPG signals with artifacts.

The trained model was tested on the test set containing a mix of clean and irrelevant PPG windows. The test set is unlabeled, so a visual assessment was needed to verify the model's efficacy. Figure 10 displays four different parts taken from the test set. The green zone is where the model predicted the signal as clean and the red zone as artifacts. From the figures it can be seen that the model can efficiently predict the irrelevant PPG windows where it is difficult to extract important features. This paper, in addition to summarizing data, proposed a data augmentation process for medical time series collected from wearable devices and sensors to increase the amount of training data and make the model more robust to noise. 

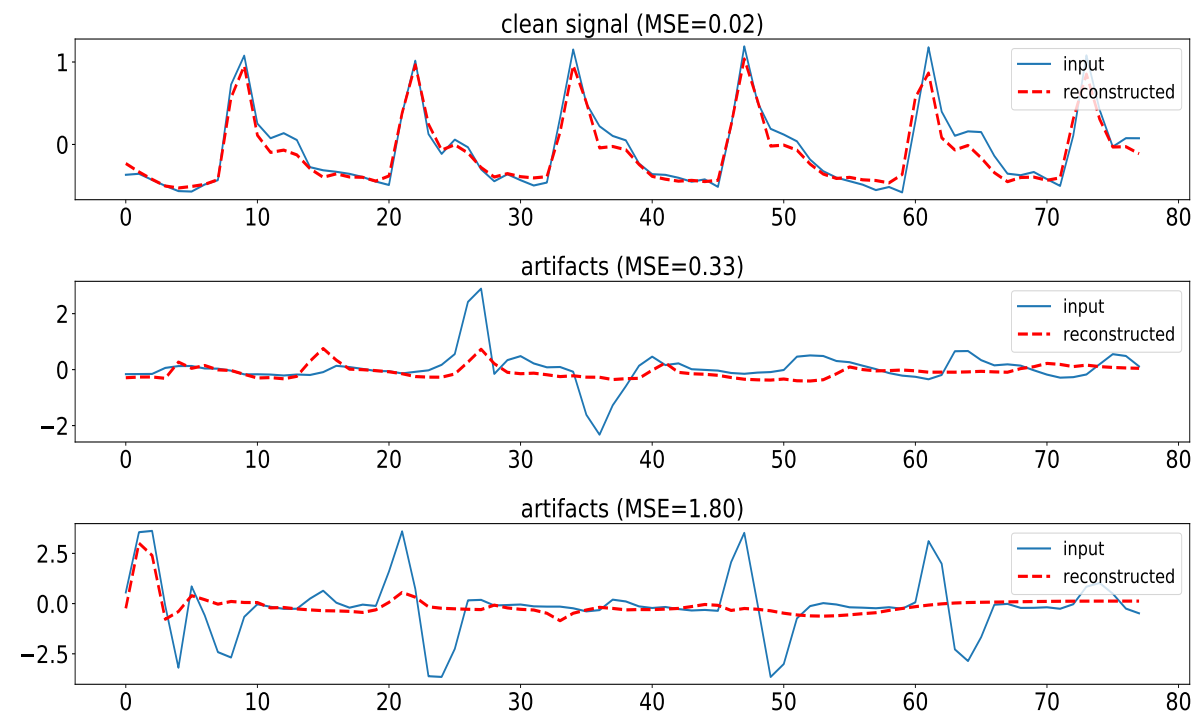

Figure 9: Examples of mean squared error obtained when clean PPG signals and artifact signals are reconstructed

\section{Conclusion}

This paper proposed a deep learning model for automatic motion artifacts detection from photoplethysmography. Before training the model, two major steps were taken that helped to achieve better results. First, a data summarization step was applied to the input data through the discrete wavelet transform to reduce the length of the sequences and avoid vanishing gradients. As a result, summarizing large windows can still allow the model to learn the same patterns while processing less data points, the model can better learn patterns from larger data windows than small ones. 


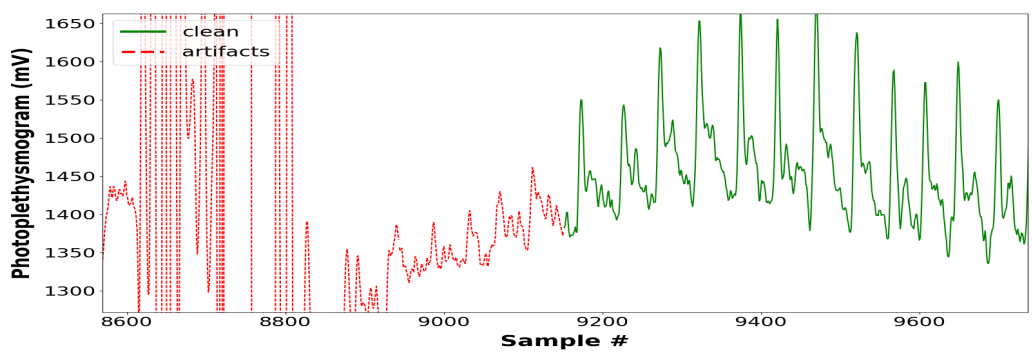

(a)

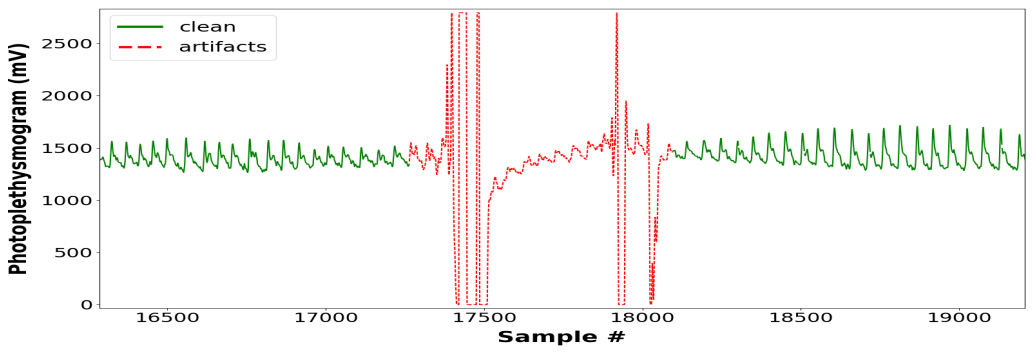

(b)

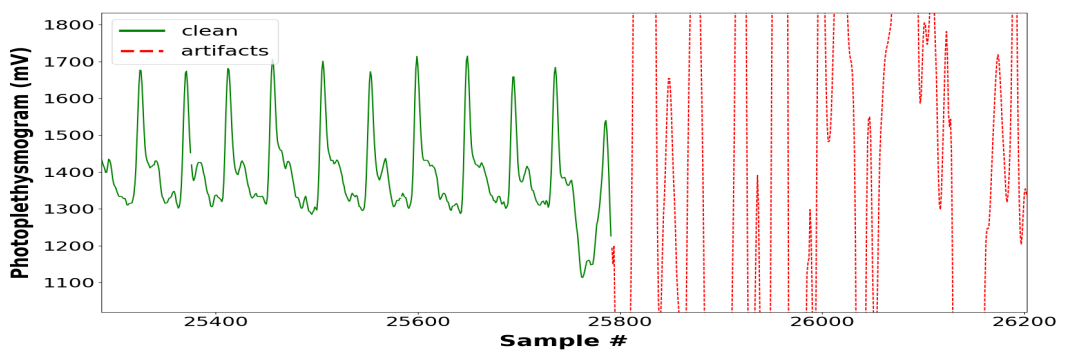

(c)

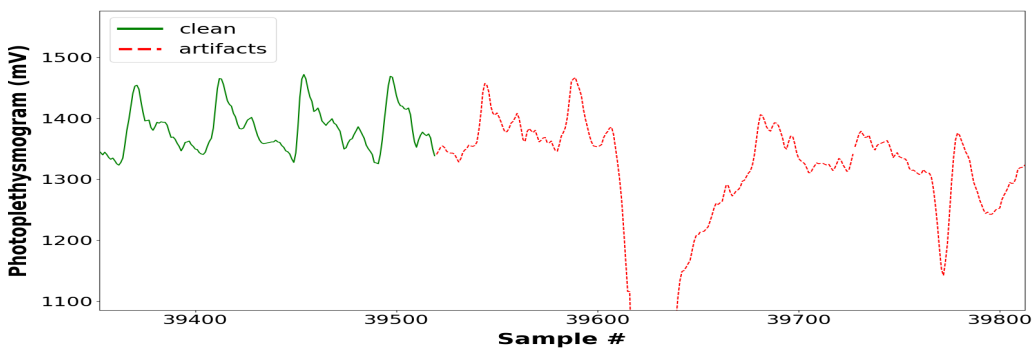

(d)

Figure 10: Four windows taken from the test set showing the prediction results obtained from the trained model 
Finally, a CNN-LSTM autoencoder architecture was proposed to detect and discard irrelevant zones in photoplethysmogram signals in order to avoid analyzing and processing meaningless data. The findings of this paper show that the proposed approach to data summarization and augmentation helped to improve the performance of the neural network and was able to achieve $90 \%$ precision and $95 \%$ recall. The proposed method in this paper can be generalized for various types of medical time series such as electrocardiogram (ECG) data in addition to periodic sensory data.

\section{Acknowledgment}

This work is partially funded with support from the Hubert Curien CEDRE programme n40283YK and the EIPHI Graduate School (contract "ANR17-EURE-0002"). Computations have been performed on the supercomputer facilities of the "Mésocentre de calcul de Franche-Comté"

\section{References}

\section{References}

[1] Sungmee Park, S. Jayaraman, Enhancing the quality of life through wearable technology, IEEE Engineering in Medicine and Biology Magazine 22 (3) (2003) 41-48. doi:10.1109/MEMB.2003.1213625.

[2] A. Pantelopoulos, N. G. Bourbakis, A survey on wearable sensor-based systems for health monitoring and prognosis, IEEE Transactions on Systems, Man, and Cybernetics, Part C (Applications and Reviews) 40 (1) (2010) 1-12. doi:10.1109/TSMCC.2009.2032660.

[3] D. Castaneda, A. E. andMohammad Ghamari, C. Soltanpur, H. Nazeran, A review on wearable photoplethysmography sensors and their potential future applications in health care, International Journal of Biosensors and Bioelectronics 4 (4) (2018) 195-202. 
[4] P. van Gent, H. Farah, N. van Nes, B. van Arem, Heartpy: A novel heart rate algorithm for the analysis of noisy signals, Transportation Research Part F: Traffic Psychology and Behaviour 66 (2019) 368 - 378. doi:https: //doi.org/10.1016/j.trf.2019.09.015.

[5] D. Kwon, H. Kim, J. Kim, S. C. Suh, I. Kim, K. J. Kim, A survey of deep learning-based network anomaly detection, Cluster Computing 22 (1) (2019) 949-961. doi:10.1007/s10586-017-1117-8.

[6] M. Elgendi, On the analysis of fingertip photoplethysmogram signals, Current Cardiology Reviews 8 (1) (2012) 14-25.

[7] P. van Gent, H. Farah, N. van Nes, B. van Arem, Analysing noisy driver physiology real-time using off-the-shelf sensors: Heart rate analysis software from the taking the fast lane project, Journal of Open Research Software 7 (1) (2018) 32.

[8] M. Singha Roy, R. Gupta, J. K. Chandra, K. Das Sharma, A. Talukdar, Improving photoplethysmographic measurements under motion artifacts using artificial neural network for personal healthcare, IEEE Transactions on Instrumentation and Measurement 67 (12) (2018) 2820-2829. doi: 10.1109/TIM. 2018.2829488

[9] G. Cennini, J. Arguel, K. Akşit, A. van Leest, Heart rate monitoring via remote photoplethysmography with motion artifacts reduction, Opt. Express 18 (5) (2010) 4867-4875. doi:10.1364/OE.18.004867.

[10] M. R. Askari, M. Rashid, M. Sevil, I. Hajizadeh, R. Brandt, S. Samadi, A. Cinar, Artifact removal from data generated by nonlinear systems: Heart rate estimation from blood volume pulse signal, Industrial \& Engineering Chemistry Research.

[11] A. Bacà, G. Biagetti, M. Camilletti, P. Crippa, L. Falaschetti, S. Orcioni, L. Rossini, D. Tonelli, C. Turchetti, Carma: A robust motion artifact reduction algorithm for heart rate monitoring from ppg signals, in: 2015 23rd 
European Signal Processing Conference (EUSIPCO), 2015, pp. 2646-2650. doi:10.1109/EUSIPCO.2015.7362864.

[12] B. S. Kim, S. K. Yoo, Motion artifact reduction in photoplethysmography using independent component analysis, IEEE Transactions on Biomedical Engineering 53 (3) (2006) 566-568. doi:10.1109/TBME. 2005.869784.

[13] B. Lee, J. Han, H. J. Baek, J. H. Shin, K. S. Park, W. J. Yi, Improved elimination of motion artifacts from a photoplethysmographic signal using a kalman smoother with simultaneous accelerometry, Physiological Measurement 31 (12) (2010) 1585-1603. doi:10.1088/0967-3334/31/12/003. URL https://doi .org/10.1088\%2F0967-3334\%2F31\%2F12\%2F003

[14] M. Raghuram, K. Venu Madhav, E. Hari Krishna, K. Ashoka Reddy, Evaluation of wavelets for reduction of motion artifacts in photoplethysmographic signals, in: 10th International Conference on Information Science, Signal Processing and their Applications (ISSPA 2010), 2010, pp. 460-463. doi:10.1109/ISSPA.2010.5605443

[15] M. R. Ram, K. V. Madhav, E. H. Krishna, N. R. Komalla, K. A. Reddy, A novel approach for motion artifact reduction in ppg signals based on as-lms adaptive filter, IEEE Transactions on Instrumentation and Measurement 61 (5) (2012) 1445-1457. doi:10.1109/TIM.2011.2175832.

[16] J. Azar, C. Habib, R. Darazi, A. Makhoul, J. Demerjian, Using adaptive sampling and dwt lifting scheme for efficient data reduction in wireless body sensor networks, in: 2018 14th International Conference on Wireless and Mobile Computing, Networking and Communications (WiMob), 2018, pp. 1-8. doi:10.1109/WiMOB.2018.8589093.

[17] J. Azar, R. Darazi, C. Habib, A. Makhoul, J. Demerjian, Using dwt lifting scheme for lossless data compression in wireless body sensor networks, in: 2018 14th International Wireless Communications Mobile Computing Conference (IWCMC), 2018, pp. 1465-1470. doi:10.1109/IWCMC.2018. 8450459 
[18] G. Tzanetakis, P. Cook, Musical genre classification of audio signals, IEEE Transactions on Speech and Audio Processing 10 (5) (2002) 293-302. doi: 10.1109/TSA.2002.800560.

[19] A. Subasi, Epileptic seizure detection using dynamic wavelet network, Expert Systems with Applications 29 (2) (2005) 343 - 355. doi:https: //doi.org/10.1016/j.eswa.2005.04.007

[20] T. T. Um, F. M. J. Pfister, D. Pichler, S. Endo, M. Lang, S. Hirche, U. Fietzek, D. Kulic, Data augmentation of wearable sensor data for parkinson's disease monitoring using convolutional neural networks, CoRR abs/1706.00527. arXiv:1706.00527.

[21] M. X. Cohen, Fundamentals of Time-Frequency Analyses in Matlab/Octave, sinc(x) Press, 2014.

[22] V. Nair, G. E. Hinton, Rectified linear units improve restricted boltzmann machines, in: Proceedings of the 27th International Conference on International Conference on Machine Learning, ICML'10, Omnipress, USA, 2010, pp. $807-814$.

[23] K. He, X. Zhang, S. Ren, J. Sun, Delving deep into rectifiers: Surpassing human-level performance on imagenet classification, CoRR abs/1502.01852. arXiv:1502.01852.

[24] D. P. Kingma, J. Ba, Adam: A method for stochastic optimization, cite arxiv:1412.6980Comment: Published as a conference paper at the 3rd International Conference for Learning Representations, San Diego, 2015 (2014).

[25] Shimmer sensing, http://www.shimmersensing.com/products/shimmer3wireless-gsr-sensor, accessed: 2019-10-30. 